\title{
PHASE SYNCHRONIZATION IN DIRECTIVE ANTENNA ARRAYS WITH PARTICULAR APPLICATION TO THE RADIO RANGE BEACON ${ }^{1}$
}

\author{
By F. G. Kear
}

\section{ABSTRACT}

With the development of the TL antenna system for use with the radio range beacon a new problem was encountered. It became necessary to provide a positive means of stabilizing the space pattern. Slight detuning of the antennas would alter the course indications. The extent to which detuning affects the pattern is demonstrated in detail and the limits to which the tuning must be maintained are shown to be very rigid. To overcome this difficulty two types of excitation systems have been developed in which the stability of the space pattern is independent of the antenna tuning to a marked degree. Either a parallel connected pair of lines $90^{\circ}$ in electrical length or a series connection of lines $180^{\circ}$ in length is shown to possess this characteristic.

Experimental data on several types of lines show the system to be practical for use along the airways, and no sacrifice of the desirable features of the TL antenna is required. Tests of the system on actual airway range beacons show it to be satisfactory.

\section{CONTENTS}

I. Introduction

II. Effect of phasing on the space pattern

III. Analysis of current and voltage relations

IV. Building out sections

1. Laboratory tests

2. Tests on the antenna system 132

3. Tests on parallel connection 133

4. Tests on series connection

5. Tuning methods

VI. Conclusions

\section{INTRODUCTION}

In order to eliminate the fluctuations of radio range-beacon courses during the hours of darkness this Bureau recently developed, for the Research Division of the Aeronautics Branch, Department of Commerce, a new type of transmitting antenna system which has been named the TL antenna. This research has been described in a recent paper. $^{2}$ Briefly, this sytem consisted of substituting for the loop antennas previously used, two pairs of vertical antennas producing the same radiated space pattern in the ground plane as that created by the loop antennas, but of which the electric field was substantially vertically polarized. It was shown that this system was practically free from night fluctuation effects and also possessed other desirable

1 The substance of this paper has been submitted to the Massachusetts Institute of Technology in partial fulfillment of the requirements of a doctorate thesis. Copies of the complete thesis will be on file in the Library of the Institute.

2 H. Diamond, The Cause and Elimination of Night Efiects in Radio Range-beacon Reception, B.S. Jour. Research, vol. 10 (RP513), p. 7, January 1933. 
features. However, the space pattern produced by these antennas was essentially unstable in that it was dependent upon all tuning adjustments remaining constant. To overcome this difficulty an excitation system has been developed which synchronizes the cur-
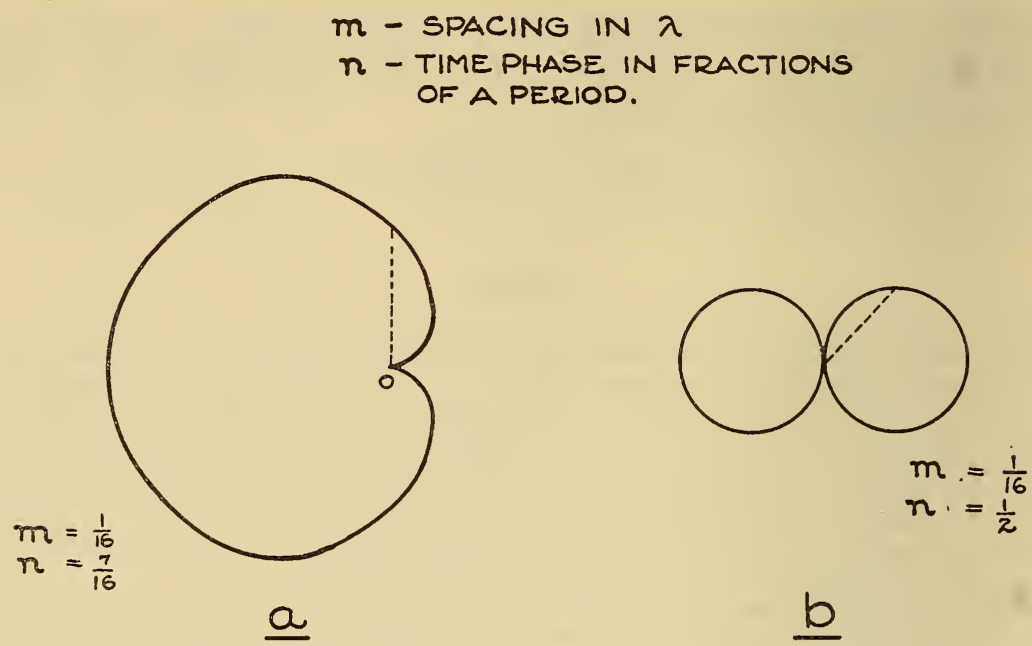

$\underline{b}$
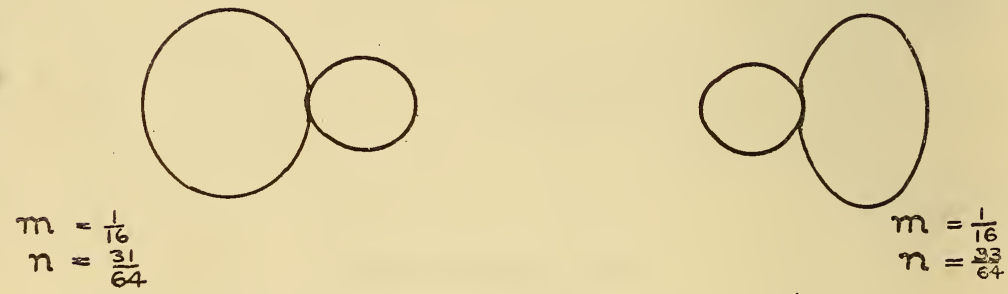

C

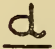

FIGURE 1.-Space palterns of two vertical antennas (various phasings).

rents in each pair of antennas to an extent which makes their stability comparable to that of the loop antenna.

\section{EFFECT OF PHASING ON THE SPACE PATTERN}

The directional characteristics of antenna arrays have been discussed at length in various papers. ${ }^{3}$ These analyses, however, 'deal chiefly with antennas whose separation is a large fraction of a wave length and of which the phasing is changed in similar fractions. In the range-beacon system the antenna separation varies from 400 to 600 feet depending upon the ground available, which corresponds to

${ }_{3}^{3}$ For example: A. M. Foster, Directive Diagrams of Antenna Arrays, Bell System Tech. J, vol. 5, p. 292, 1926. 
possible separations of from one eleventh to one sixth wave length for the frequency band employed. In addition to this, the so-called "on course" zone is created by the intersection of two independent space patterns. These two factors require that the phase of the currents be kept within much closer limits than is necessary with the customary directional array.

The general equation for the space pattern of two antennas in the ground plane is

$$
E=K[\cos (\pi m \cos \theta+\pi n)]
$$

(where only the terms necessary for indicating the pattern are included). $E$ is the intensity at constant radius and at an angle of $\theta$ degrees from

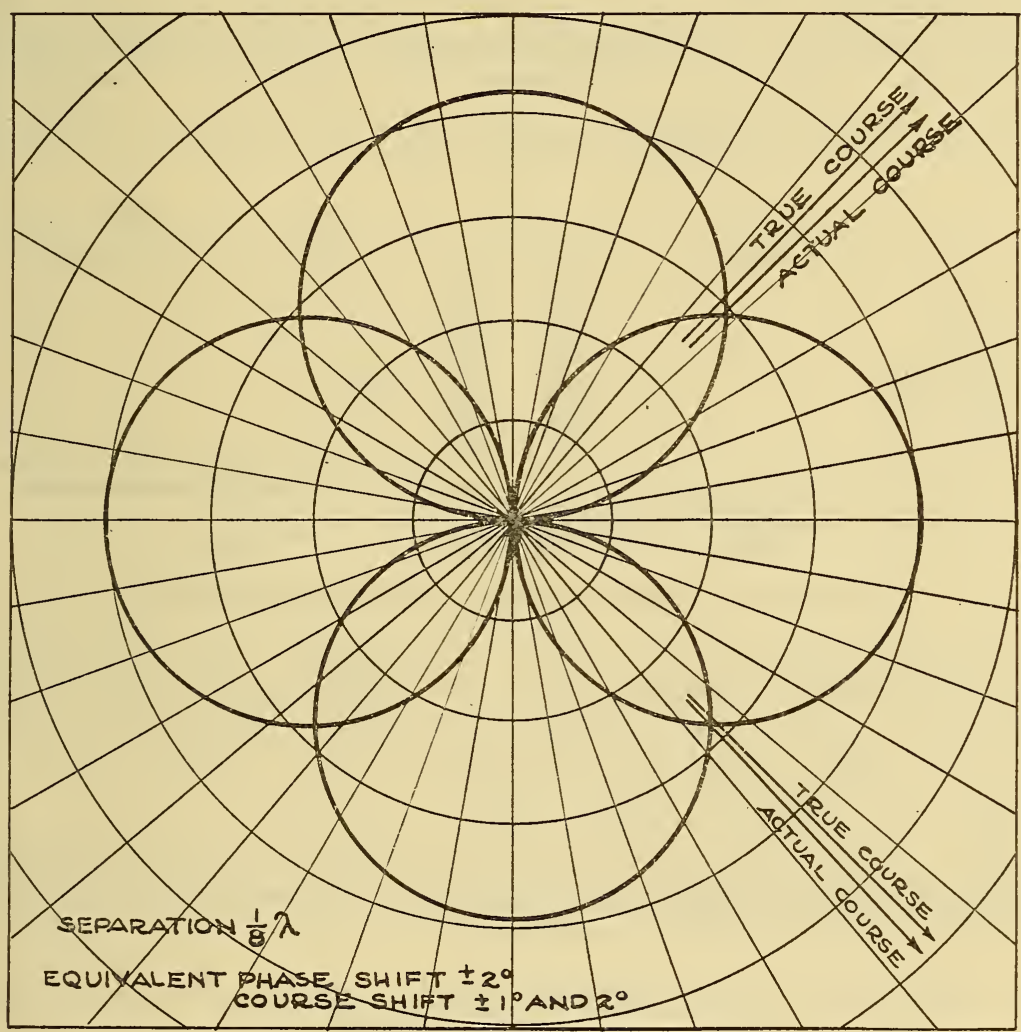

FIGURE 2.-Effect of slight phase shift on space pattern.

an arbitrary zero. $m$ is the spacing of the two antennas in wave lengths or fractions thereof while $n$ is the time phase in fractions of a period. Two sets of phase relations are commonly employed with such an antenna group. The first is $m+n=1 / 2$ which results in a cardioid pattern. The second is $n=1 / 2$ and $m<1 / 2$. This gives the figure-of-eight pattern. These are illustrated in figure $1(a)$ and $(b)$.

In range-beacon work the figure-of-eight is most commonly employed with some slight change in the value of $n$ to permit course bending. This variation is described in footnote 2. For a change of phase from the correct value the pattern is altered considerably. 
Figure $1(c)$ and $(d)$ show the extent of this change in the figure-ofeight pattern for phase variations of $5^{\circ}$, and for antennas spaced one sixteenth wave length. This is the spacing of the antennas at the College Park range beacon It is obvious that a change of this magnitude will not be allowable.

Since some change of phase will undoubtedly occur because of changing antenna constants, it is necessary to determine within what limits such changes are permissible. Figure 2 shows a space pattern for the range beacon with a $2^{\circ}$ change of phase of one antenna. This shifts the course indication from $1^{\circ}$ to $2^{\circ}$, which is as much as can be tolerated. A phase shift of $2^{\circ}$ was therefore selected as the maximum permissible shift.

A total shift of $2^{\circ}$ may be caused by very slight changes in the circuit constants. Consider, for example, the cage antennas used at Bellefonte. These had a resistance of $10 \mathrm{ohms}$ and a capacity of $500 \mu \mu \mathrm{f}$. The phase angle between current and voltage is $\operatorname{arc} \tan \frac{X}{R}$.

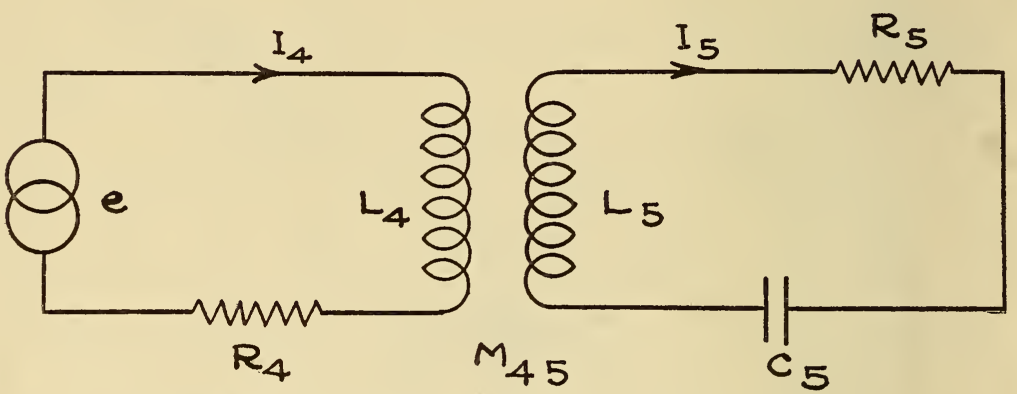

Figure 3.-Equivalent circuit of coupling transformer.

At resonance this is zero. For $\theta$ to be equal to $2^{\circ}, \tan \theta=0.035$. Assuming matched impedances in the exciting circuit the effective resistance in the antenna circuit is $20 \mathrm{ohms}$. This means a value of $X=0.7 \mathrm{ohm}$. Now $X=\left|X_{L}-X_{c}\right|$ and if we assume $X_{L}$ to be constant, then $\Delta X_{c}=0.7 \mathrm{ohm}$.

This corresponds to a change of only $1 \mu \mu \mathrm{f}$ at the frequencies employed. A cage antanna or even a steel tower cannot be expected to remain constant within these limits so in consequence some means of synchronization must be employed.

\section{ANALYSIS OF CURRENT AND VOLTAGE RELATIONS}

The first step toward securing a suitable circuit was the design of a coupling system which had maximum inherent stability. The transmission lines used with the TL antenna consisted of a pair of rubbercovered wires surrounded by a metallic sheath. It had a low surge impedance and required impedance matching transformers at each end. (The line characteristics are discussed in detail later.) Instead of using coupling transformers, such as are frequently adopted for radio circuits, and matching impedances by securing coupling conditions of $\omega^{2} M^{2}=R_{1} R_{2}$, transformers with a very high degree of coupling and high self-inductance were employed. It can readily be shown that if the $\frac{\omega L}{R}$ of each coil is very large compared to the load imped- 
ances and if the coupling coefficient is high, a transformer will act purely as an impedance-matching device in which the ratio of transformation (turns ratio) is equal to the square root of the ratio of impedances to be matched. For example, consider the circuit of figure 3. We may write the following relations:

whence

$$
\begin{aligned}
& e=Z_{4} I_{4}+Z_{45} I_{5} \\
& O=Z_{45} I_{4}+Z_{5} I_{5}
\end{aligned}
$$

but

$$
\begin{aligned}
& I_{4}=\frac{Z_{5} e}{Z_{4} Z_{5}-Z_{45}{ }^{2}} \\
& I_{5}=-\frac{Z_{45} e}{Z_{4} Z_{5}-Z_{45}{ }^{2}} \\
& \frac{I_{5}}{I_{4}}=-\frac{Z_{45}}{Z_{5}}=-\frac{j \omega M_{45}}{R_{5}+j \omega L_{5}}
\end{aligned}
$$

$$
j \omega L_{5} \gg R_{5}
$$

$$
\begin{aligned}
& L_{4}=K n_{4}{ }^{2} \\
& L_{5}=K n_{5}{ }^{2}
\end{aligned}
$$

where $K$ is a geometric constant

$$
M_{45}=C \sqrt{L_{4} L_{5}}=C \sqrt{\bar{K}^{2} n_{4}{ }^{2} n_{5}^{2}}=C K n_{4} n_{5}
$$

where $C$ is the coefficient of coupling.

$$
\begin{aligned}
\frac{I_{5}}{I_{4}} & =-\frac{j \omega M_{45}}{j \omega L_{5}} \\
& =-\frac{M_{45}}{L_{5}}=-\frac{C K n_{4} n_{5}}{K n_{5}{ }^{2}} \cong-\frac{n_{4}}{n_{5}}(\text { as } C \cong 1)
\end{aligned}
$$

Under these conditions, therefore, the current in the secondary is related to that in the primary solely by means of the turns ratio. Furthermore

$$
\begin{aligned}
I_{4} & =\frac{\left(R_{5}+j X_{5}\right) e}{\left(R_{4}+j X_{4}\right)\left(R_{5}+j X_{5}\right)+X_{45}{ }^{2}} \\
& =\frac{e}{\left(R_{4}+j X_{4}\right)+\frac{X_{45}{ }^{2}}{\left(R_{5}+j X_{5}\right)}} \\
& =\frac{e}{\left(R_{4}+j X_{4}\right)+\frac{X_{45}{ }^{2}}{R_{5}{ }^{2}+X_{5}{ }^{2}}\left(R_{5}-j X_{5}\right)}
\end{aligned}
$$


where

$$
=\frac{e}{\left(R+\varphi R_{5}\right)+j\left(X_{4}-\varphi X_{5}\right)}
$$

$$
\begin{aligned}
\phi & =\frac{X_{45}{ }^{2}}{R_{5}{ }^{2}+X_{5}^{2}} \text { and since } X_{5}^{2} \gg R_{5}^{2} \\
& =\frac{X_{45}{ }^{2}}{X_{5}^{2}} \\
& =\frac{M_{45}{ }^{2}}{L_{5}^{2}}=\frac{\left(C K n_{4} n_{5}\right)^{2}}{\left(K n_{5}\right)^{2}}=\frac{C^{2} n_{4}^{2}}{n_{5}{ }^{2}} \cong \frac{n_{4}{ }^{2}}{n_{5}^{2}}
\end{aligned}
$$

The impedances therefore are reflected through the circuit by a constant proportional to the square of the turns ratio. Now should the coefficient of coupling be unity $(C=1)$

and

$$
\begin{aligned}
\phi X_{5} & =\frac{X_{45}{ }^{2}}{X_{5}{ }^{2}} \times X_{5} \\
& =\frac{X_{45}{ }^{2}}{X_{5}} \cong \frac{K^{2} n_{4}{ }^{2} n_{5}{ }^{2}}{K n_{5}{ }^{2}} \\
& =K n_{4}{ }^{2}=X_{4}
\end{aligned}
$$

$$
j\left(X_{4}-\phi X_{5}\right)=j\left(X_{4}-X_{4}\right)=0
$$

So for unity coupling the inductances do not affect the circuit. Should the coupling be less than unity there will remain some of the inductive terms which can be removed by making $X_{5}$ (or $X_{4}$ ) capacitative to the amount of such discrepancy. For high coefficients of coupling (90 percent is secured on the coils in actual use) the ratio of transformation is not altered greatly and is compensated for by the method of tuning. This analysis neglects the effect of capacity coupling between circuits. For the lines in use the voltages and frequencies were low enough so that the capacity could be neglected. The resulting circuit is shown in figure $4(a)$. By replacing the transformers with their equivalent leakage reactance the circuit now simplifies to that of figure $4(b)$. The series circuit at the end of each line is much easier to deal with in considering synchronization than would be a parallel circuit.

Since the relation between primary and secondary currents in the transformer is fixed, if the transformer primary currents in two opposite lines are synchronized the antenna currents will also be synchronized. That allows the circuit under consideration to be simplified still further to that of figure $4(c)$. When this simplification is made, there is at once apparent a very simple method of attack. Consider the ratio of sending end voltage to receiving end current; that is, the transfer impedance. This is

$$
Z_{T R}=\frac{E_{S}}{I_{R}}=Z_{R} \cosh \gamma l+Z_{o} \sinh \gamma l^{4}
$$

where $\gamma$ is the propagation constant of the line per unit length, $Z_{\sigma}$ the surge impedance and $Z_{R}$ the load impedance.

\footnotetext{
- Everitt, communication engineering, McGraw Hill Co. (book).
} 
Now, in general, a change of $Z_{R}$ will result in a change of $Z_{T R}$. However, if cosh $\gamma l=0, Z_{T R}$ is independent of the load, and the current-voltage relation is determined solely by $Z_{0}$. For a line with negligible attenuation $\cosh \gamma l \cong \cos \beta l$, a circular function which is zero for $\beta l=$ any odd multiple of $\frac{\pi}{2}$. Consequently, if the line is 90 electrical degrees long and has a low attenuation, $Z_{T R}=j Z_{0}$ and the load current will bear a constant relation to the sending voltage.

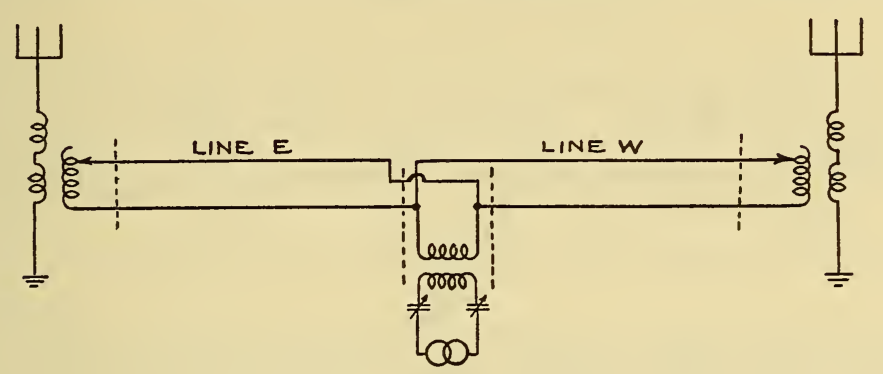

a
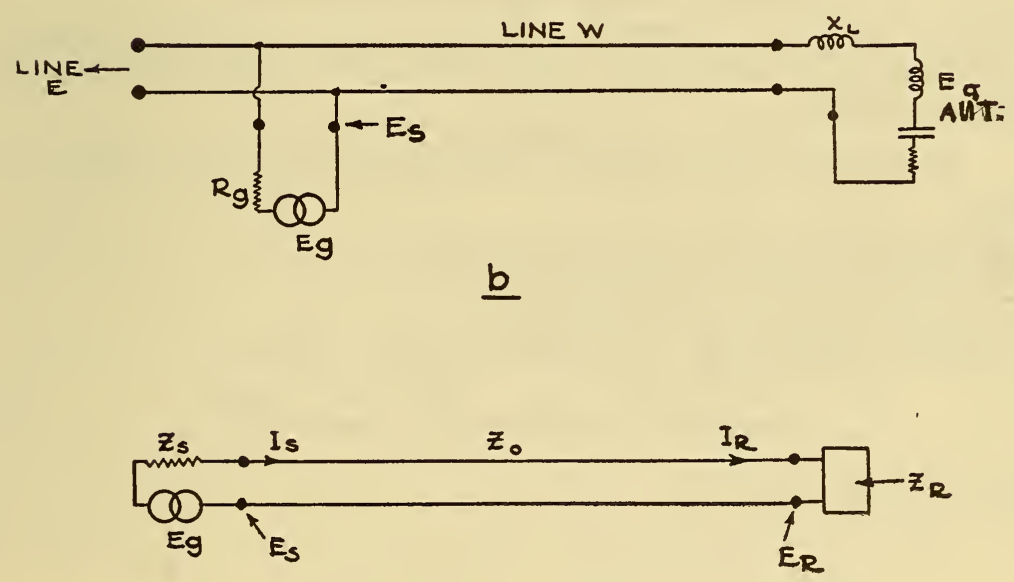

c

FIgURE 4.-Antennas excited by parallel connections.

$a$, actual circuit; $b$, first simplification; and $c$, final circuit analyzed.

Since this applies to each line supplied from $E_{S}$, the relation of $I_{R 1}$ to $I_{R 2}$ is constant regardless of antenna tuning. $I_{R 1}$ will, of course, change for a change of $Z_{R 1}$, but at the same time $E_{S}$ is constrained to follow such a change and $I_{R 2}$ is changed likewise. The result is total synchronization of the two currents.

There is also an alternative method of attack. The loop antennas are free from phase shift, since the two sides of the loop form a continuous series circuit. We may similarly connect the transmission lines in series as in figure $5(a)$. The hybrid coil at $E_{S}$ is employed 


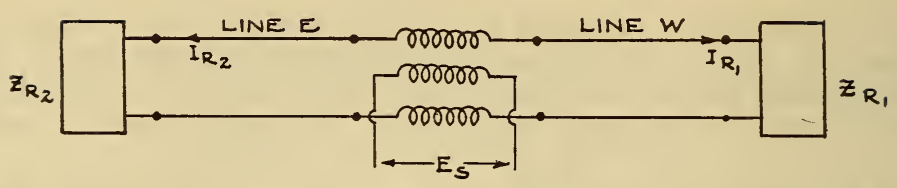

a

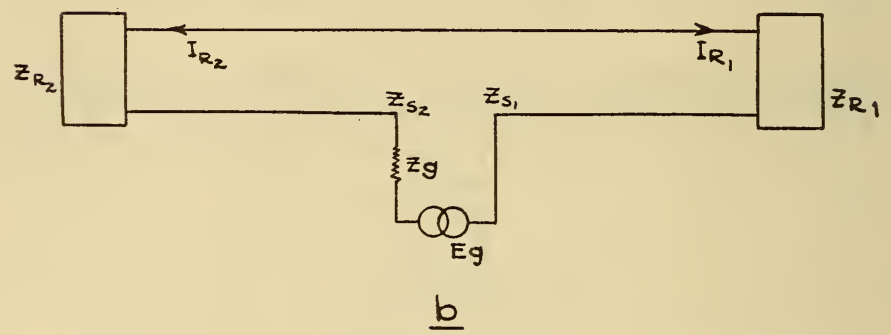

FigURE 5.-Antennas excited by a series connection.

$a$, actual circuit; and $b$, equivalent circuit analyzed.

merely to balance the voltage applied to the lines and prevent an unbalance of sheath currents. For purposes of analysis it may be considered as in figure $5(b)$. For such a circuit the current is given by the equation:

where

$$
I_{S 1}=\frac{E_{g}\left[Z_{o} \cosh \gamma e+Z_{R 1} \sinh \gamma e\right]}{Z_{o}\left(Z_{R 1}+Z_{S 2}+Z_{g}\right) \cosh \gamma e+\left[Z_{o}^{2}+Z_{R 1}\left(Z_{S 2}+Z_{g}\right)\right] \sinh \gamma e}
$$

$Z_{o}$ is the surge impedance of the line

$Z_{S 2}$ is the input impedance of line 2

$Z_{g}$ is the generator impedance.

Assume antenna 2 to be properly tuned. Then

$$
Z_{S_{2}}=R_{S_{2}} Z_{g}=R_{g} \text { and } R_{g}+R_{S_{2}}=Z_{S}^{\prime} \underline{0}^{0}
$$

We may now write

$$
\begin{aligned}
I_{R_{1}} & =\frac{E_{g}}{\left(Z_{R}+Z_{S}\right) \cosh \gamma l+\left(Z_{o}+\frac{Z_{R} Z_{S}}{Z_{o}}\right) \sinh \gamma l} \\
& =\frac{\mathrm{E}_{g} Z_{o}}{Z_{o}\left(Z_{R}+Z_{S}\right) \cosh \gamma l+\left(Z_{o}^{2}+Z_{R} Z_{S}\right) \sinh \gamma} l
\end{aligned}
$$

and sincc $I_{S_{1}}=I_{S_{2}}=I_{g}$

$$
\begin{aligned}
& \frac{I_{S}}{I_{R_{1}}}=\frac{Z_{o} \cosh \gamma l+Z_{R_{1}} \sinh \gamma l}{Z_{o}} \\
& \frac{I_{S}}{I_{R_{1}}}=\cosh \gamma l+\frac{Z_{R_{1}}}{Z_{o}} \sinh \gamma l
\end{aligned}
$$


For the line which is properly terminated

$$
\frac{I_{S}}{I_{R_{2}}}=\cosh \gamma l+\sinh \gamma l
$$

Hence

$$
\frac{I_{R_{1}}}{I_{R_{2}}}=\frac{\cosh \gamma l+\sinh \gamma l}{\cosh \gamma l+\frac{Z_{R_{1}}}{Z_{o}} \sinh \gamma l}
$$

That is to say, the phase and magnitude of $I_{R_{1}}$ will change with respect to $I_{R_{2}}$ as antenna 1 is detuned. Now if $\sinh \gamma l<<\cosh$ $\gamma l$ this change will be negligible. This means a very short line (a simple series circuit) or again if the attenuation is small, any line which is a multiple of $\frac{\lambda}{2}$ in length, since under that condition $\sinh \gamma l \cong$ $j \sin \beta l=0$.

For half wave lines connected in series we may, therefore, expect synchronization of the two antenna currents. Both the quarter and the half wave lines were tested experimentally to determine how closely they would conform to the theory.

\section{BUILDING-OUT SECTIONS}

As has previously been stated, the separation of the antennas in the airway installations varies from 400 to 600 feet. From the exciting transformer to the antenna, therefore, is usually less than a quarter wave length considering the phase velocities of the lines used. It would be possible to use sufficient line to make the length a quarter or a half wave length as desired and coil the portion in excess of the actual length at the base of each antenna. This is quite expensive, however, and also inflexible should a change in transmitting frequency be made. To avoid this, the lines were cut to the length required to reach the antennas and were then built out to the desired length by artificial lines within the range-beacon house. These building-out sections were designed for the same propagation constant and surge impedance as the actual lines so that no discontinuities were introduced. Furthermore, the low voltage and current on the lines permitted small compact units occupying much less space than the actual cable. One such section is illustrated in figure 6 .

\section{EXPERIMENTAL WORK}

\section{LABORATORY TESTS}

First tests on this method of synchronizing were made in the laboratory where control of the circuit parameters was possible. Two types of transmission line were available, the first consisted of two strands of no. 14 rubber-covered wire in an enclosing lead sheath and the second two no. 12 rubber-covered wires within first a lead and next a steel sheath (Parkway cable). Since the first was easier to handle it was used for most of the tests although the greatly reduced losses in the Parkway cable made its use desirable in the final arrangement. The characteristics of these lines will be found in the appendix. 
Using two sections of line each 120 feet long and connected in series (see fig. 7), the change of phase of receiving end currents was observed as a load on the end of one line was changed. This observation was made on a cathode-ray oscillograph and a shift of $5^{\circ}$ from the correct

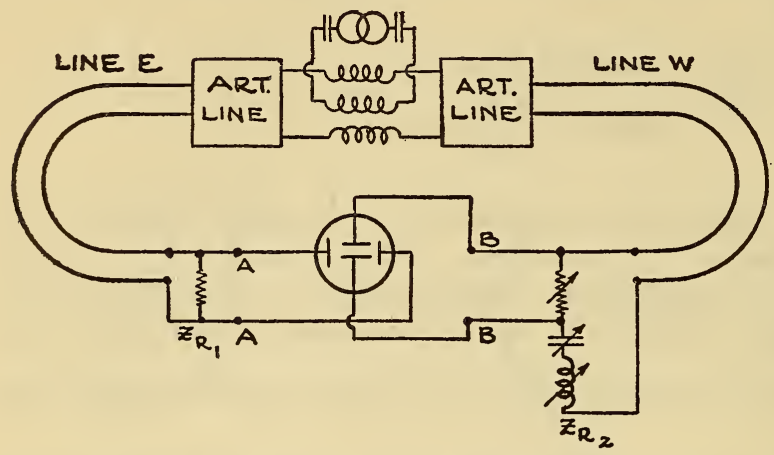

FIGURE 7.-Circuit for oscillographic study. phase could readily be observed. These lines were approximately $40^{\circ}$ long and no synchronization was noticed. They were then built out with suitable sections to half-wave lines and further observations made. It was now possible to vary $Z_{R}$ from the correct value of $75 \mid 0^{\circ}$ to $120 \mid 33^{\circ}$ and $120 \mid \overline{10}$ without losing synchronization. The fact that beyond these limits synchronizing action was lost was ascribed to the relatively high loss of this type of line.

A similar set of tests was made with a parallel connection of the lines built out to a quarter wave length. The results were about the same; if anything, somewhat better. It was therefore concluded to try the same circuits on an actual antenna system.

\section{TESTS ON THE ANTENNA SYSTEM}

There were at College Park two pairs of vertical antennas 40 feet high and separated 220 feet from each other. These were excited by means of the transmission lines built out to various lengths and the synchronizing action studied. The coupling unit is illustrated in figure 8 , which shows the compact nature of the arrangement.

To determine the degree of synchronization two antennas were excited, and the voltage induced in a third antenna, equidistant from the other two, was measured by means of a milliammeter inserted in its base. If the phase is correct and the currents in the antennas are equal there will be no current apparent in the third antenna.

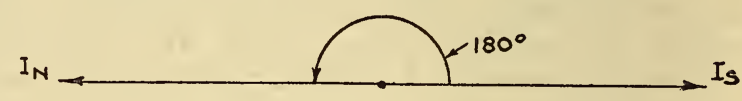

However, a slight unbalance of magnitude or phase will cause the milliammeter to indicate. For example, if each antenna independently $\left(I_{n}\right.$ and $I_{s}$ in fig. 9 ) causes $100 \mathrm{ma}$ of current to flow in the test antenna, then a phase difference of $2^{\circ}$ from the true $180^{\circ}$ relationship will cause a current of 3 ma when both antennas are excited $\left(I_{R}\right.$ in fig. 9$)$. These 
B.S. Journal of Research, RP581

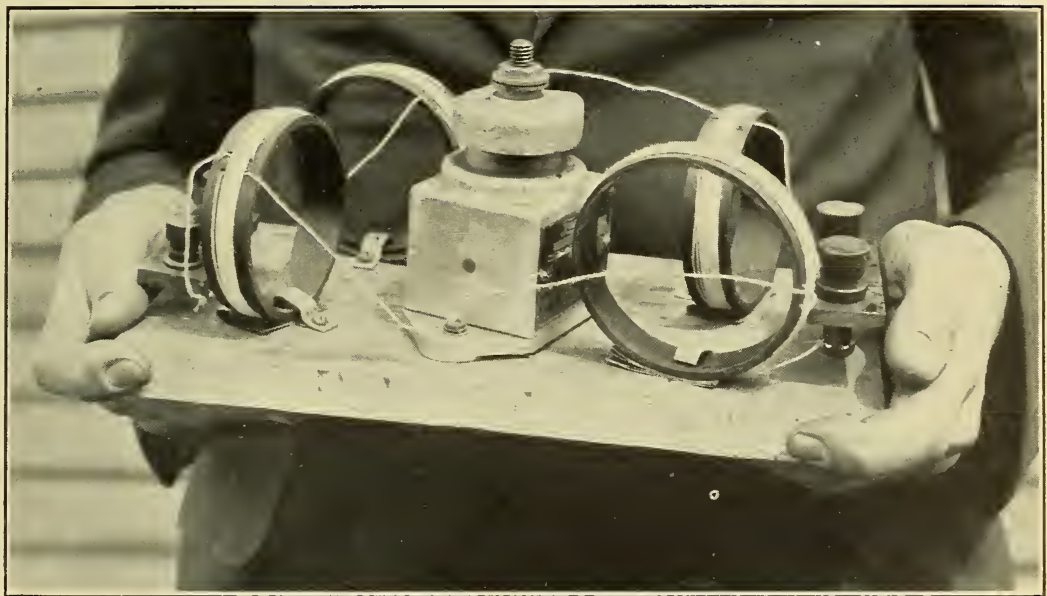

Figure 6.-Building-out section. 
B.S. Journal of Research, RP581

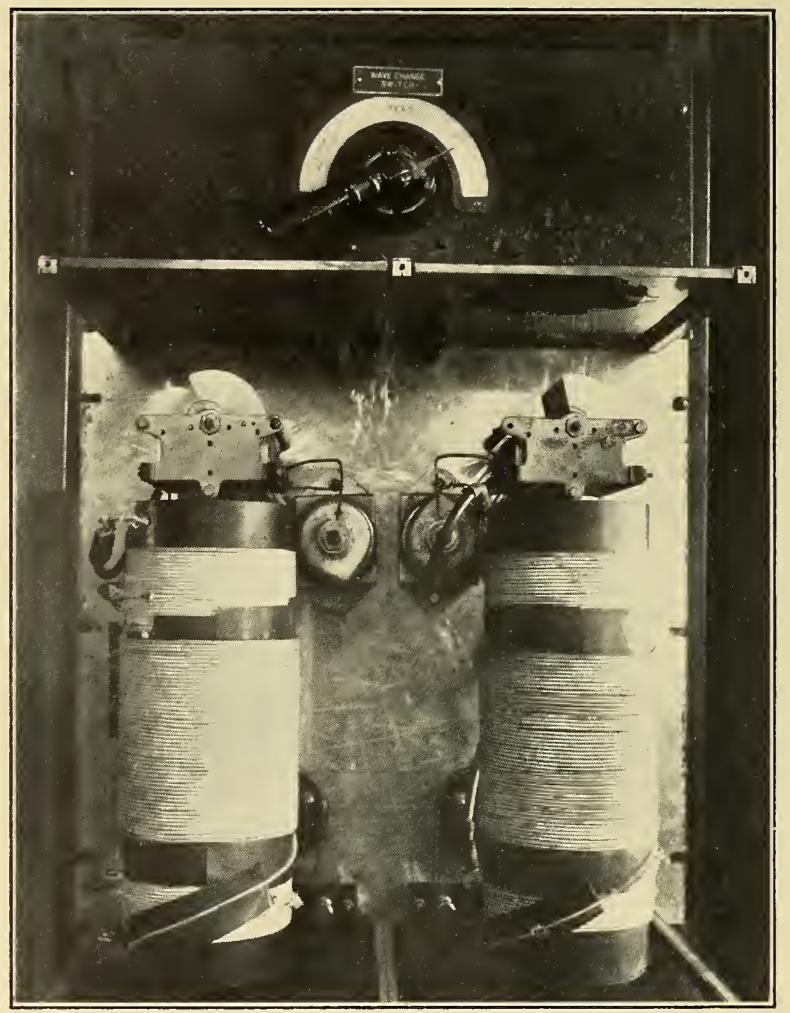

FIgURE 8.-Coupling unit with matching transformers. 
currents could easily be read on the meter employed and since $2^{\circ}$ is the variation which is chosen for the maximum allowable deviation, the method of observation was adequate. Previous experience with this method of adjustment had also shown it to be satisfactory. ${ }^{5}$

\section{TESTS ON PARALLEL CONNECTION}

With the parallel connection of figure 4 , a series of tests were made with various line lengths. The graph of figure 10 gives a comparison between the lines formerly in use $\left(40^{\circ}\right)$ and the $90^{\circ}$ lines. With the former the instability was very serious. Slight swinging of the antenna in the wind caused large changes in phase. With the $90^{\circ}$ lines the antennas could not only be detuned until the reactance was \pm 15 percent of the antenna resistance without dephasing, but even when definitely beyond the zone of control no changing of phase was

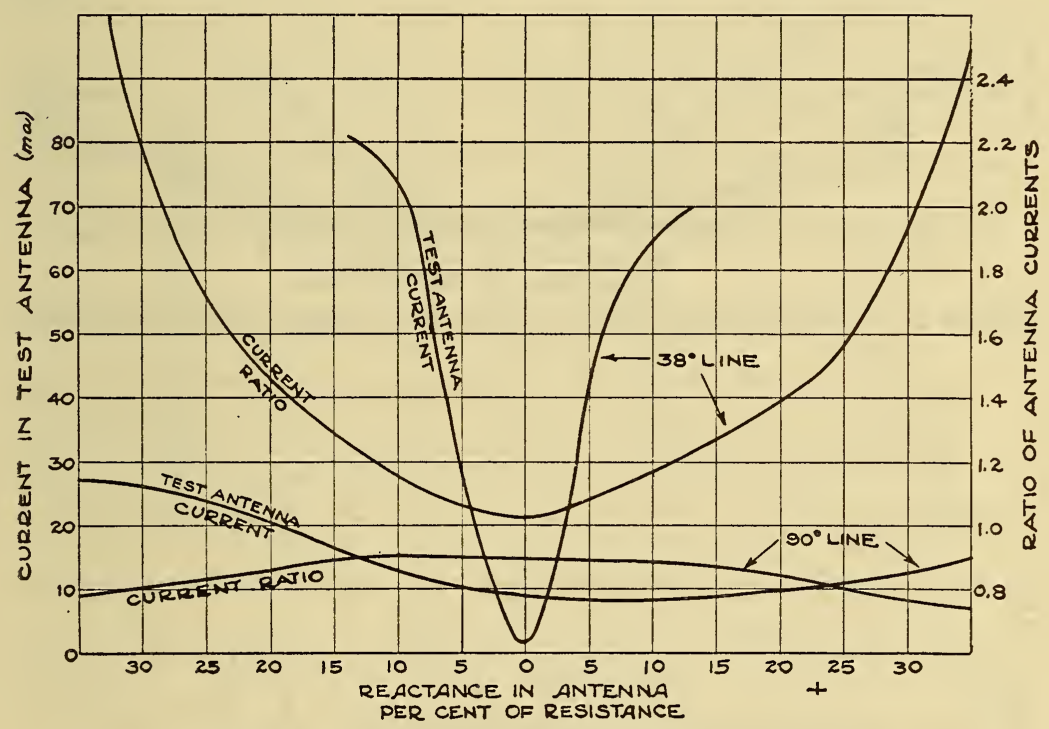

FIGURE 10.-Synchronization data (lines in parallel).

observed with a swinging antenna. In figure 10 the current flowing in the third antenna (the test antenna) is plotted against the amount of detuning of one of the antennas of the array with the other antenna in correct tune. For the $38^{\circ}$ line a change of less than 2 percent in reactance caused a change of current of more than 3 ma. For the $90^{\circ}$ line a change of reactance of \pm 15 percent was necessary before the test antenna current changed $3 \mathrm{ma}$. Unfortunately in this test the ratio of currents in the antennas of the array did not equal unity, consequently there was no zero current condition but merely a minimum. The ratio of antenna currents plotted on the same graph indicates the existence of this condition. The data of figure 11, and those which follow it, were secured with an equality of currents in the two antennas under test, consequently the curves go through a zero rather than a minimum.

B See footnote 2, p. 123. 
Tests were next conducted to determine the optimum line length and the allowable deviation from correct length. Figures 11 and 12 give the results of these tests. It will be noted that the optimum length is

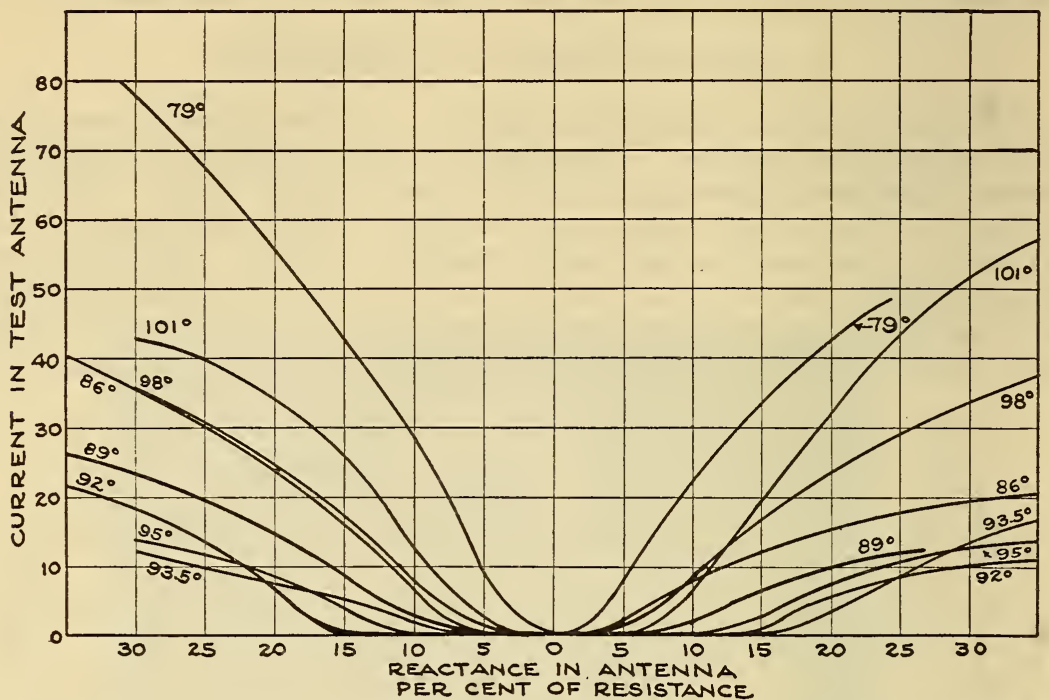

FIGURE 11.-Synchronizing data for various line lengths (parallel connection).

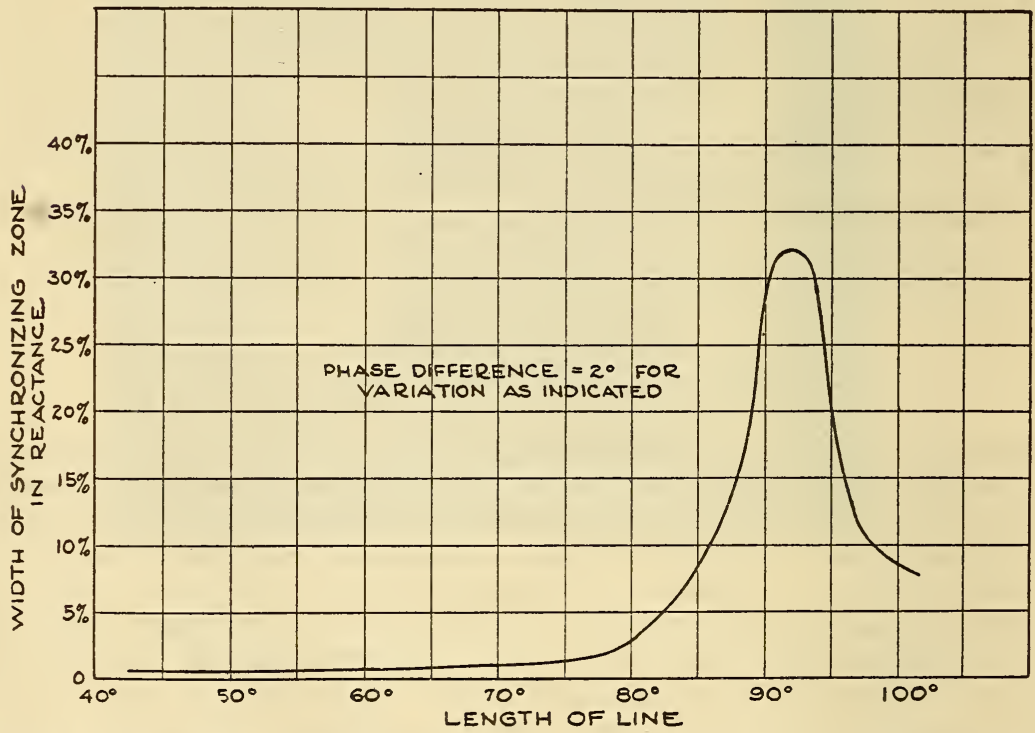

FIGURE 12.-Width of synchronizing zone as a function of line length (parallel connection).

$93^{\circ}$ and not $90^{\circ}$. It is apparent that the exact length is not important. Several degrees difference is possible with good synchronism. This means that the north line may be $98^{\circ}$ and the south line $88^{\circ}$, with the resultant antenna currents synchronizing at $170^{\circ}$ instead of $180^{\circ}$. 
As a result of this feature the method of course bending described in footnote 2 may be applied without sacrificing the action of the lines in maintaining the pattern.

Further analysis of the data used in plotting figure 11 reveals another interesting fact. Figure 11 was plotted so that the curves are all approximately symmetrical about an arbitrary zero reactance point. As a matter of fact the location of the minimum shifts with line lengths. Lines which are greater than the optimum length have a minimum in the negative reactance side of tuning. Lines which are short have their minimum in the positive reactance side. The location of these minima with respect to line length is plotted in figure 13 . In adjusting the line length it can immediately be determined whether the line under test is too long or too short by merely noting the center of maximum synchronization with respect to resonance, and referring

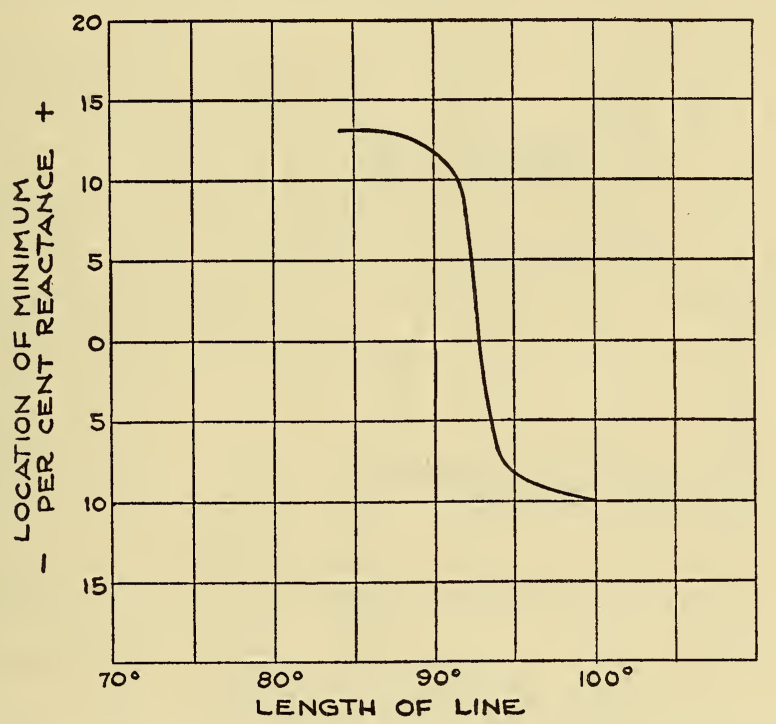

FIGURE 13.-Location of minimum versus line length (parallel connection).

to the curve. This is of great assistance in the installation of such a system where it is desired to use the maximum possible synchronization.

A final set of curves is given in figure 14 for the case of lines with very low attenuation. This is approximately the type of line used on the airways and the range of synchronizing action makes the resulting: antenna system comparable in stability with the loop antennas. The antenna currents in the north and south antennas are plotted on this graph as a function of the amount of detuning. The relative values of these currents are of interest in that when synchronization is secured the currents rise and fall together. With no synchronization the reverse is true, making the space pattern depart even further from the desired shape.

Two other details were investigated. A line with nonuniform structure was substituted for the uniform line. About half of the line had a surge impedance of $75 \mathrm{ohms}$ and the remainder $55 \mathrm{ohms}$. This had no apparent effect upon the synchronizing action. The effect of mismatch 
of the antenna to the line which was similar in nature was also studied. It was found that any tuning adjustment which roughly matched the impedances within plus or minus 15 percent, operated very well. From these results it is safe to say that the system is decidedly noncritical in its action and consequently field installations can be made under adverse conditions with reasonable certainty of successful operation.

\section{TESTS ON SERIES CONNECTION}

Tests similar to those made for the parallel connection were made for the series case. The action was in accord with the theory, when allowance was made for the loss due to relatively high attenuation. The maximum amount of detuning permissible was then \pm 12.5 percent as compared with \pm 16 percent for similar lines connected in parallel.

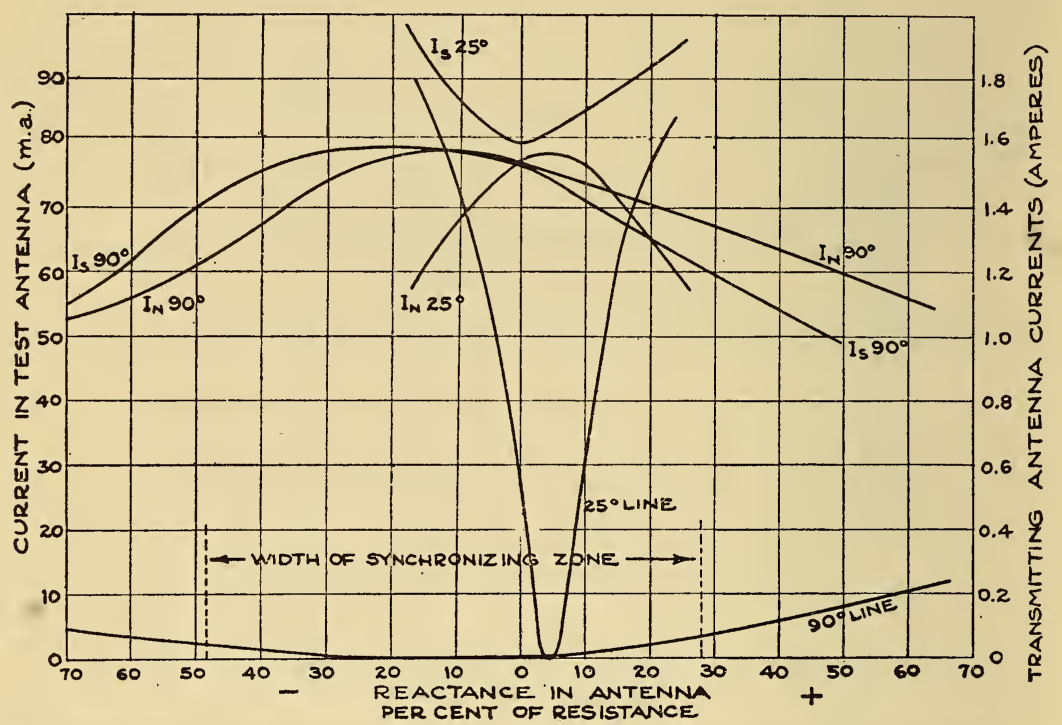

FIGURE 14.-Synchronizing data. Low loss lines. (Parallel connection.)

The increased loss because of greater line length no doubt caused some of this reduction. Furthermore, with the series connection the current is essentially of a circulating nature through the two antennas. Because of this the effect of sheath capacity would be more serious than in the parallel connection. Open wire lines would probably be preferable for series use.

Figures 15 and 16 show graphically the action of the series lines. They should be compared with figures 11 and 12 of the parallel line curves, since the same type of line was used in the two cases. The parallel connection seems to be preferable, as it operates over a wider range, requires less line length, and has a simpler exciting circuit. However, both methods are satisfactory. For use in the airway installations the $90^{\circ}$ line has been adopted as a standard. 


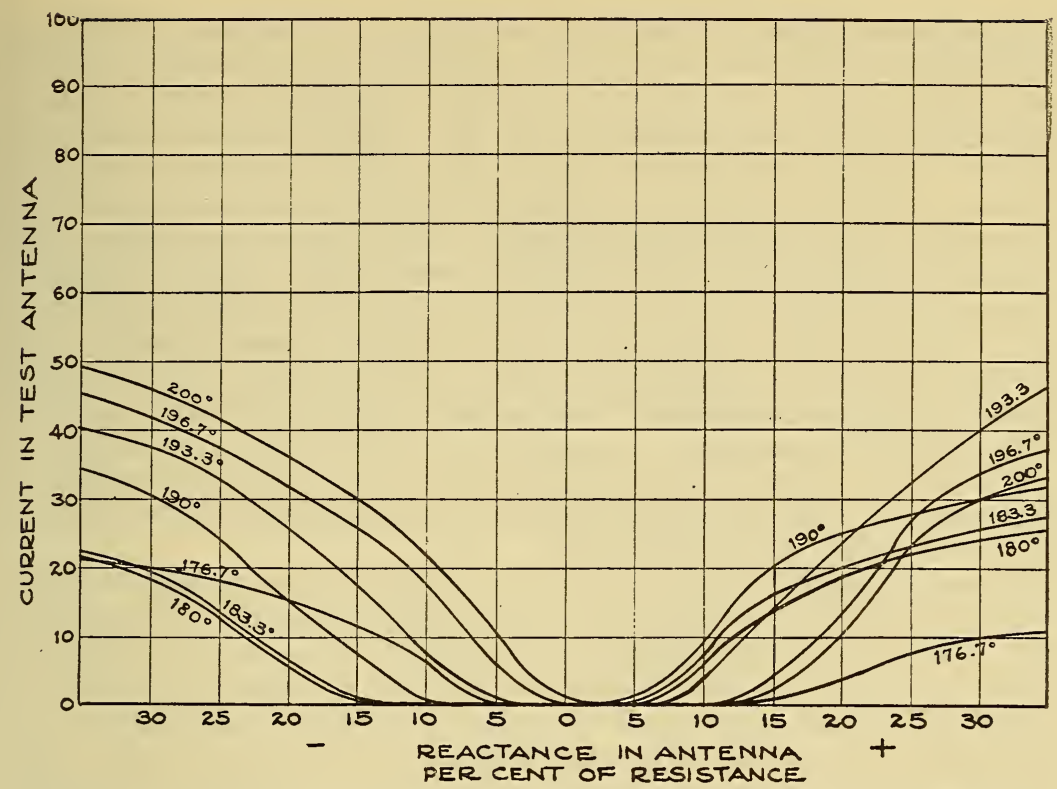

FIGURE 15.-Synchronizing data for various line lengths (series connection).

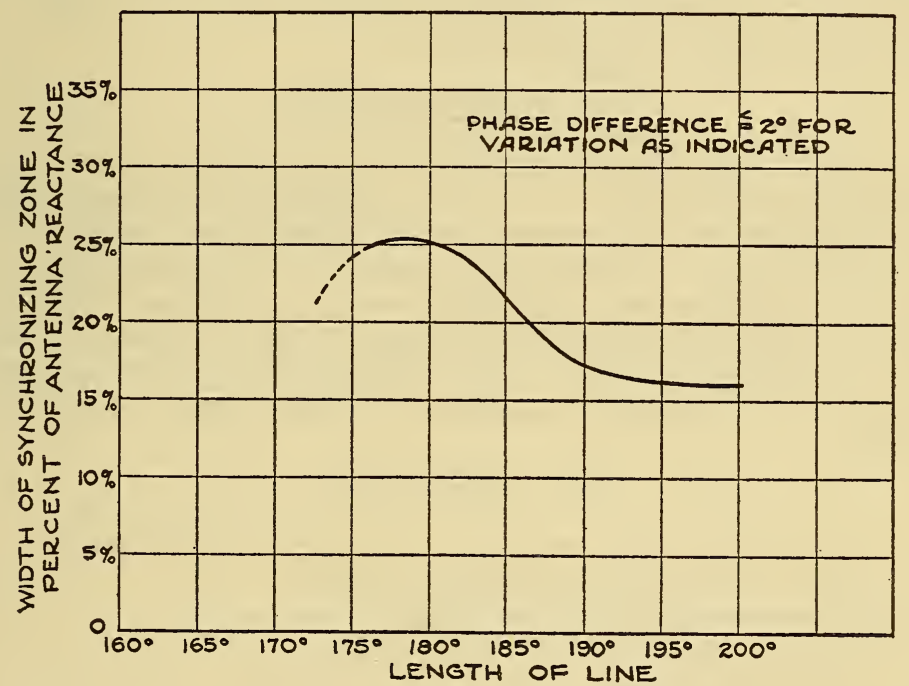

FIGURE 16.-Width of synchronizing zone as a function of line length (series connection).

\section{TUNING METHODS}

Since the tuning adjustments are no longer critical, almost any uniform procedure may be adopted. At College Park the procedure was as follows: Three lines were terminated in their surge impedance; that is, a noninductive resistor of the proper value. The line being adjusted was first terminated in its impedance and the current into 
the impedance noted. It was then connected to the antenna through the coupling transformer and the antenna tuning adjusted for maximum line current. If this was greater than the current into the termination the transformation ratio was increased and the antenna retuned. If the current was lower, the ratio was decreased. The ratio was adjusted until the maximum line current just equaled the current into the terminating resistor. The antenna was then tuned for maximum antenna current, which meant only a slight misadjustment and increased the field intensity appreciably. Because of the synchronizing action this did not disturb the space pattern. Each antenna in turn was adjusted and then all four lines were connected to their respective antennas. No further readjustment was necessary.

Where course bending is necessary, the line lengths are calculated to provide the proper phase relationship and the tuning is carried out in the same manner as just described. For the manner of determining. the proper phase see footnote 2.

Because of capacity between the transformer primary and secondary the currents in the two wires of the line were not equal. This made no difference as far as operation was concerned. However, by inserting in the lead from the transformer to ground a condenser of which the reactance equaled the negative of half the leakage reactance of the transformer, the nodal point was shifted to the center of the transformer and the currents balanced. With such a condenser the meter readings of the currents into the four antennas all balance and it is an easier matter to check the operation of the system as a whole. It is an added refinement but not necessary.

\section{CONCLUSIONS}

The results of the experimental work show the two types of line to operate very satisfactorily. The $90^{\circ}$ parallel line has been tested in actual service for several months and provides a very satisfactory and stable space pattern. It is simple to calculate and install and it makes tuning of the antennas a straightforward procedure. The further extension of this synchronizing principle to other types of antennas presents no great difficulty. With the present trend to directional antennas on all frequency channels it should find a wide field of usefulness.

\section{APPENDIX}

Transmission line constants measured at $290 \mathrm{kc} / \mathrm{sec}$.

1. 2-wire no. 14 rubber-covered lead sheath:

Surge impedance $Z_{o}=53.5 \mid 0^{\circ} \mathrm{ohm}$.

$\gamma=0.00138+j 0.00577$ per foot of cable.

$\alpha=0.138$ per 100 feet.

$\beta=33^{\circ}$ per 100 feet.

Phase velocity, 59,400 miles/sec.

Attenuation, $3.76 \mathrm{db}$ per 100 feet. 
2. Parkway cable no. 12 rubber-covered wire, lead and steel sheaths:

Surge impedance $Z_{0}=690^{\circ}$.

$\gamma=0.00014+j 0.00362$.

$\beta=20.7^{\circ}$ per 100 feet.

$\alpha=0.014$ per 100 feet.

Phase velocity, 92,800 miles/sec.

Building-out sections (computed for $290 \mathrm{kc} / \mathrm{sec}$.).

Parallel connection, Parkway cable.

Length of line $24^{\circ} 40^{\prime}$,

Length of section $65^{\circ} 20^{\prime}$ (for $90^{\circ}$ line).

$Z_{1}=Z_{2}=Z_{o} \tanh \frac{\theta}{2}=j 69 \tan 32^{\circ} 40^{\prime}$ (attenuation neglected) $=j 44.6$ ohm.

$L_{1}=L_{2}=25.2 \mu \mathrm{h}$.

Each coil of $\mathrm{H}$-section is then $12.6 \mu$ h (fig. 17).

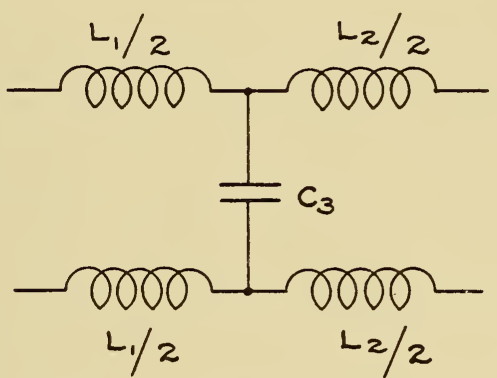

Figdre 17.-Equivalent $\mathrm{H}$-section.

$$
\begin{aligned}
Z_{3} & =\frac{Z_{0}}{\sinh \theta} \cong-j \frac{70}{\sin 65^{\circ} 20^{\prime}} \\
& =-j 77.2 \mathrm{ohm} . \\
C_{3} & =0.007_{3} \mu \mathrm{f}
\end{aligned}
$$

Series connection. Lead cable.

For 115 feet of line $\beta l=38^{\circ}$.

Length of section $=142^{\circ}$ for $180^{\circ}$ line.

$Z_{1}=Z_{2}=Z_{0} \tan h \frac{\theta}{2}$

$=j 54 \tan 71^{\circ}$.

$=j 156.8 \mathrm{ohms}$.

$L_{1}=L_{2}=81.9 \mu \mathrm{h}$

Each coil of $\mathrm{H}$-section $=41 . \mu \mathrm{h}$.

$$
\begin{aligned}
Z_{3} & =\frac{Z_{0}}{\sinh \theta} \cong-j \frac{54}{\sin 142^{\circ}} \\
& =-j 87.7 \mathrm{ohm} . \\
C_{3} & =0.0063 \mu \mathrm{f} .
\end{aligned}
$$

Other sections were computed similarly.

Washington, May 12, 1933. 